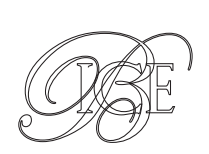

\title{
CANADÁ: UN PAÍS DE OPORTUNIDADES PARA LA INVERSIÓN EXTRANJERA EN AGROALIMENTACIÓN, TECNOLOGÍAS LIMPIAS Y TRANSPORTE
}

Canadá ofrece un sinfín de oportunidades para las empresas españolas que buscan crecer, expandirse a nuevos mercados e invertir a escala internacional. Este artículo proporciona una descripción general de tres sectores canadienses: el agroalimentario, el de tecnologías limpias y el de transporte, donde Canadá ha alcanzado un liderazgo global y ofrece una amplia gama de oportunidades de inversión. Explica cómo las cadenas de suministro integradas de Canadá, sus acuerdos de libre comercio y los incentivos gubernamentales progresivos convierten al país en una de las mejores opciones para los inversores globales. El artículo concluye describiendo otros factores que hacen de Canadá una ubicación privilegiada para las empresas globales que planean iniciar o expandir sus operaciones internacionales.

Palabras clave: Canadá, sector agroalimentario, energías limpias, incentivos para inversores.

Clasificación JEL: O31, O51.

\section{La elección de Canadá como destino de inversión}

Empresas de todo el mundo eligen Canadá como destino de inversión. Numerosas empresas españolas han expandido ya sus negocios en este país. Atraídas por una economía diversa y estable y un entorno empresarial favorable, las empresas globales descubren rápidamente que las sofisticadas cadenas de suministro canadienses incluyen todo lo que precisan para ayudar a sus

\footnotetext{
* Jefa de Servicios para Inversores, Invest in Canada. Versión de agosto de 2021.

DOI: https://doi.org/10.32796/bice.2021.3140.7279
}

negocios a expandirse y prosperar en los mercados internacionales.

\subsection{Materias primas y talento cualificado}

Conocido mundialmente como un país rico en recursos naturales, que incluyen vegetales, minerales y metales, bosques y vida marina, Canadá ofrece mucho más que materias primas. Las cadenas de suministro canadienses combinan fabricación avanzada y tecnologías innovadoras.

Canadá es el hogar de la fuerza laboral con nivel de formación más elevado del mundo, y ocupa el primer lugar entre los países de la $\triangle$ 
OCDE por proporción de titulados superiores, graduados en universidades o colegios universitarios (colleges), entre la población en edad de trabajar (OECD, 2020). Aproximadamente el $24 \%$ de las matriculaciones postsecundarias se registran en ciencias, tecnología, ingeniería y matemáticas (Statistics Canada, 2021) y, además, Canadá cuenta con un elevado número de colegios universitarios y universidades acreditadas a escala mundial. Los trabajadores y profesionales altamente cualificados de Canadá son innovadores y diligentes. Poseen el conocimiento técnico necesario para introducir productos innovadores en los mercados de todo el mundo.

Canadá también atrae talento global a través de iniciativas como la Estrategia Global de Habilidades (Global Skills Strategy), que ha traído al país a más de 40.000 trabajadores cualificados desde 2017 (Immigration, Refugees and Citizenship Canada, 2019). La estrategia ofrece tres vías para que los trabajadores cualificados accedan a Canadá: exenciones para permisos de trabajo de corta duración, resolución de las solicitudes para talento de alta cualificación en tan solo dos semanas y la Vía Talento Global (Global Talent Stream), que está diseñada para las empresas innovadoras de Canadá que necesitan extranjeros únicos y especializados para poder escalar y crecer (Employment and Social Development Canada, 2021a).

\subsection{Acuerdos comerciales}

Canadá ha impulsado activamente acuerdos de libre comercio en todo el mundo con el fin de abrir las puertas a nuevos mercados para las empresas y los inversores canadienses. Entre los países del G7, Canadá es el único que mantiene acuerdos comerciales con todos y cada uno de los restantes miembros del G7. A través de catorce acuerdos comerciales con 51 países, Canadá brinda a los inversores globales acceso a casi 1.500 millones de consumidores en todo el mundo. La proximidad geográfica del país y el acuerdo de libre comercio con Estados Unidos ofrecen a los inversores globales acceso a la mayor economía del mundo (Invest in Canada, 2021).

De interés directo para las empresas españolas, el Acuerdo Económico y Comercial Integral entre Canadá y Europa (CETA) elimina los aranceles sobre el $98 \%$ de las líneas arancelarias con la Unión Europea en casi todos los sectores. EI CETA también facilita el movimiento de mercancías y profesionales capacitados a través de las fronteras e incluye un proceso para reconocer rápidamente las certificaciones profesionales. Las disposiciones sobre inversiones del CETA están diseñadas para ofrecer a los inversores mayor certeza, estabilidad y protección para su inversión y contemplan el acceso a un mecanismo independiente de resolución de disputas (Global Affairs Canada, 2020).

\subsection{Incentivos y programas gubernamentales}

Canadá ha realizado importantes inversiones en innovación y crecimiento mediante el establecimiento de programas de apoyo al progreso como la Estrategia Pancanadiense de Inteligencia Artificial (Pan-Canandian Artificial Intelligence Strategy) y la Iniciativa de Superclústeres de Innovación (Innovation Superclusters Inititiative), que reúnen a la industria, las incubadoras, las universidades, los centros de investigación y los laboratorios de innovación $D$ 
con el fin de ayudar a posicionar a las empresas ubicadas en Canadá en la vanguardia de sus sectores. Estas oportunidades de colaboración impulsan la innovación en Canadá.

Centrado en adoptar un enfoque proactivo hacia la inversión extranjera, Canadá ha introducido una serie de programas y créditos fiscales para fomentar la innovación y apoyar la inversión. Con la tasa impositiva efectiva marginal más baja de todas las naciones del G7 (13,7\%), Canadá es una opción atractiva para los inversores de Europa y del resto del mundo (Department of Finance Canada, 2019). Además, las provincias y territorios del país ofrecen generosos incentivos fiscales para que los inversores se establezcan o amplíen sus operaciones en Canadá.

En este artículo se presenta un conjunto de incentivos y programas específicos para los sectores agroalimentario, de tecnologías limpias y de transporte. Muchas de estas oportunidades para inversores son aplicables a varios sectores. Incluyen:

- El Incentivo a la Inversión Acelerada (Accelerated Investment Incentive), que permite a las empresas amortizar de forma inmediata una mayor proporción del coste de los activos de capital recién adquiridos. Posibilita una desgravación de costes de capital reforzada (CCA) en la compra de equipos, como la maquinaria y el equipo utilizado para inversiones en energía limpia o para fabricar y procesar bienes.

- El Programa de Investigación Científica y Desarrollo Experimental (Scientific Research and Experimental Development, SR\&ED) proporciona créditos fiscales y reembolsos por gastos en las actividades de investigación y desarrollo elegibles realizadas en Canadá. Cada año, el programa aporta más de 3.000 millones de dólares en incentivos fiscales en todos los sectores. Estos incentivos incluyen deducciones fiscales, créditos fiscales a la inversión y reembolsos.

- El Fondo de Innovación Estratégica (Strategic Innovation Fund, SIF) ofrece financiación para apoyar la innovación en las industrias líderes de Canadá, incluidos 8.000 millones de dólares dedicados al Fondo Acelerador Cero Neto (Net Zero Accelerator Fund), que ayudará a impulsar los proyectos de descarbonización de grandes emisores, ampliar la tecnología limpia y acelerar la transformación industrial en Canadá.

\section{El agroalimentario canadiense: un terreno fértil para las proteínas de origen vegetal}

\subsection{Visión general}

El sector agroalimentario canadiense incluye cárnicos, lácteos, cereales, alimentos procesados y una sofisticada cadena de suministro que se extiende por todo el país. La agroalimentación es el sector manufacturero más grande de Canadá en términos de empleo y producto interior bruto (PIB), y emplea a 2,3 millones de canadienses (Agriculture and AgriFood Canada, 2020).

Abundante en recursos de tierra y agua, con una sólida capacidad de investigación y desarrollo y una reputación mundial consolidada como proveedor de alimentos seguros y de alta calidad, Canadá es el quinto mayor exportador de alimentos del mundo (Export Development Canada, 2019). También es el mayor productor $\square$ 
mundial de canola, trigo duro, linaza y legumbres (Government of Saskatchewan, 2020).

El ecosistema agroindustrial integrado de Canadá se basó en los principios clave de seguridad y accesibilidad. Resiliente y adaptable, la cadena de suministro agroalimentario de Canadá tiene un probado historial de logística internacional sofisticada. Gracias a amplios acuerdos comerciales, Canadá puede facilitar el movimiento de productos a través de la cadena de suministro global de agronegocios (KPMG, 2020).

Para los inversores, el sector agrícola y agroalimentario de Canadá proporciona acceso a los mercados más grandes del mundo. Entre las empresas españolas que operan en

Cascajares de España ha dominado la técnica tradicional de al vacío, que implica la cocción lenta a baja temperatura de los alimentos en envases sellados al vacío. La compañía inició operaciones en Canadá en 2009 como una forma de expandirse en el mercado norteamericano.

Después de participar en una de las incubadoras de empresas del Gobierno de Canadá, comenzaron sus operaciones en 2009 en Saint-Hyacinthe, Quebec, y recientemente se han expandido a una nueva instalación de $204 \mathrm{~m}^{2}$ y 1,5 millones de dólares (Government of Canada, 2019).

Vemos Canadá como un mercado estratégico y prioritario en el futuro del grupo, una palanca para poder vender en todo el continente americano. Por eso decidimos seguir invirtiendo, no solo en capacidad y equipamiento industrial, sino también en recursos humanos para fortalecer nuestro equipo canadiense, de manera que sigamos ejerciendo un papel de liderazgo en materia de innovación en nuestro sector. (Alfonso Jiménez Rodríguez-Vila, presidente de Cascajares, 2019)
Canadá, en el sector agroalimentario, se encuentran Cascajares, Redondo Iglesias, el Grupo Ebro y Natra Chocolate.

\subsection{Las oportunidades en el sector agroalimentario canadiense}

\section{La cadena de suministro integrada}

La avanzada cadena de suministro agroalimentario de Canadá incluye investigación y desarrollo, maquinaria, biogenética, fertilizantes y suministro de semillas. Se clasifica entre las principales oportunidades para la inversión extranjera directa después de la COVID-19, $\square$

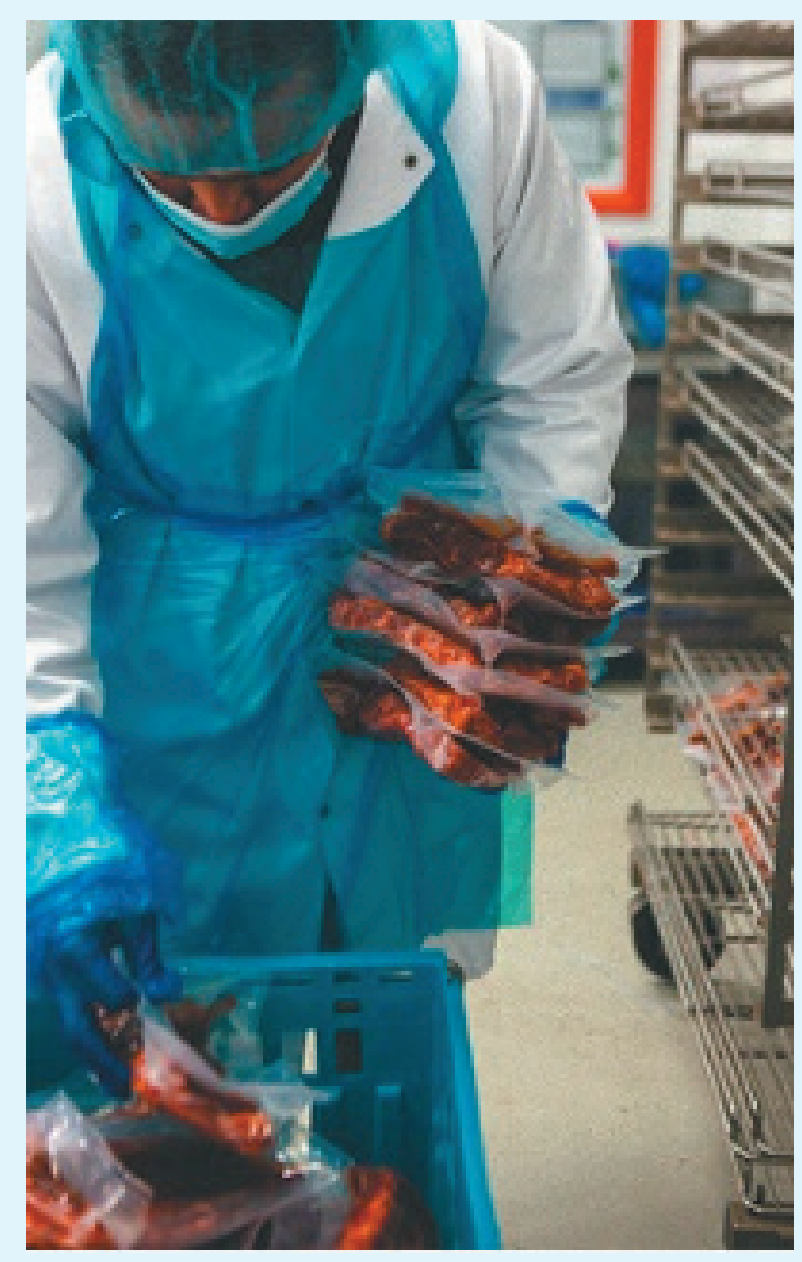

Instantánea: Cascajares. 
según el informe de KPMG de 2020 encargado por Invest in Canada. El informe revela que la estabilidad y fiabilidad de la cadena de suministro mantiene una probada resiliencia frente a los ciclos económicos y las recesiones. Además, el acceso y la proximidad de Canadá a los mercados globales ayudarán a acortar las cadenas de suministro y a aproximar los productos a sus consumidores finales, lo que resulta especialmente oportuno para las empresas españolas que pretendan expandirse por las Américas.

Es más, hay oportunidades en el horizonte para las empresas innovadoras en Canadá. El país se beneficia de una sólida reputación mundial en la producción de alimentos de alta calidad. Se espera que surjan nuevas oportunidades en el envasado y trazabilidad de los productos agroalimentarios a medida que la seguridad alimentaria se convierta en un punto focal para los consumidores globales, en especial en un entorno pospandémico.

\section{Proteínas de origen vegetal}

En el sector, de fuerte crecimiento, de las proteínas vegetales, Canadá se ha posicionado como un líder mundial. Las ventas mundiales de alternativas a la carne basadas en vegetales han crecido un promedio del $8 \%$ al año desde 2010 (Biospringer by Lesaffre, 2021). Este aumento de la demanda ha dado lugar a inversiones sin precedentes en innovación en la industria agroalimentaria.

En su calidad de mayor exportador de legumbres, Canadá es un actor clave en el mercado de alternativas de origen vegetal a la carne, que se valoró en 18.500 millones de dólares en 2019 y se espera que alcance un importe de 40.600 millones de dólares para 2025 (Research and Markets, 2019). A medida que la industria se expanda, Canadá seguirá desempeñando un papel destacado, gracias a su trayectoria acreditada de adopción de prácticas estrictas de garantía de calidad y a su compromiso continuo con la innovación.

Tecnología agraria y agricultura de precisión

Los productores agroalimentarios de Canadá utilizan la tecnología agraria (ag-tech) y la agricultura de precisión para desarrollar semillas de calidad superior, obtener más cosechas con mejor rendimiento y reducir el uso de recursos. Por ejemplo, los cultivos de legumbres reducen el impacto ambiental al fijar nitrógeno en el suelo y la atmósfera y favorecen las cosechas ulteriores. Mediante la adición de tecnología agrícola a la mezcla, estos cultivos pueden reducir en mayor medida su huella de carbono y contribuir al objetivo de Canadá de convertirse en un país carbono neutral, con emisiones netas cero para 2050.

La provincia de Alberta está a la vanguardia del desarrollo y la adopción de la tecnología agraria, mediante el uso de sistemas GPS para reducir los desechos, el monitoreo de la erosión y la provisión de una mejor gestión de los cultivos a los agricultores.

Con las nuevas soluciones de tecnología agraria surgirán nuevas oportunidades para mejorar la industria agroalimentaria de Canadá, cumplir con los objetivos ambientales globales, mejorar la seguridad alimentaria y suministrar alimentos a la población mundial, que se espera alcance los 10.000 millones en 2050.

\subsection{Los incentivos para inversores en el sector agroalimentario canadiense}

Además de una serie de programas de financiación de la innovación y de la investigación y el desarrollo, Canadá ofrece incentivos y $\triangleright$ 
dispone de programas para fomentar la inversión en el sector agroalimentario.

- El superclúster de Industrias de la Proteína de Canadá (Protein Industries Canada) reúne empresas, personas, recursos y tecnología para diversificar la cadena de valor agroalimentaria de Canadá y mejorar la tecnología de procesamiento, la nutrición vegetal y las soluciones digitales. Con sede en las provincias de las praderas de Canadá, el proyecto proporcionará hasta 153 millones de dólares en fondos y se espera que cree más de 4.500 puestos de trabajo en diez años.

- La Asociación Agrícola Canadiense (Canadian Agricultural Partnership) es una iniciativa a cinco años y de 3.000 millones de dólares para fortalecer y hacer crecer el sector de la agricultura y agroalimentación. La iniciativa está diseñada para que las empresas de la industria gestionen los riesgos, con énfasis en la innovación y el crecimiento.

\section{Las tecnologías limpias en Canadá: el impulso a la transición hacia las energías limpias}

\subsection{Visión general}

Canadá es la cuna de más de 850 empresas de tecnología limpia que trabajan para reducir las emisiones de carbono en el sector energético utilizando tecnologías innovadoras (Export Development Canada, 2020). Entre ellas se incluyen tecnologías eólicas, solares e hidráulicas, hidrógeno y pilas de combustible, y captura, utilización y almacenamiento de carbono.
Las Administraciones canadienses, en todos los niveles, están comprometidas en la lucha contra el cambio climático y la inversión en tecnologías limpias. La cadena de suministro de tecnología limpia de Canadá tiene una acreditada experiencia en logística y dispone de infraestructuras para facilitar las exportaciones.

Canadá se sitúa en la segunda posición del mundo y la primera del G20 en tecnologías limpias, según el Índice Global de Innovación de Tecnología Limpia 2021 (Canada Action, 2021). Las exportaciones de tecnologías limpias de las empresas de tecnología canadienses totalizaron 7,8 miles de millones de dólares en 2019. El sector representó el 3,2\% del PIB de Canadá y mantuvo 317.000 puestos de trabajo en 2018 (Export Development Canada, 2020).

Canadá es el segundo mayor productor de energía hidroeléctrica del mundo y es un líder reconocido en la industria del hidrógeno. El país también dispone de abundante energía eólica, hídrica y solar, y de existencias de gas natural y litio.

Con un $67 \%$ de la electricidad procedente de fuentes renovables y un $82 \%$ de fuentes que no emiten gases de efecto invernadero, Canadá se sitúa a la vanguardia de la investigación y el desarrollo colaborativo en energía renovable. El país también es líder en el desarrollo de procesos de captura, utilización y almacenamiento de carbono, con varios proyectos importantes en todo el territorio (Innovation, Science and Economic Development Canada, 2018).

En el sector de las tecnologías limpias, Canadá acoge a las empresas españolas, como ACCIONA, Enerfin, PRODESA e Iberdrola, y a otras empresas globales, como RWE, con sede en Alemania; Copenhagen Infrastructure Partners, Mercedes-Benz Canada y Samsung Renewable Energy. 
Con sede en Madrid, ACCIONA es un suministrador líder de infraestructura sostenible y de productos de energía renovable. La empresa está presente en Canadá desde 2001, donde ha completado la construcción de tres grandes puentes y 89 kilómetros de carreteras e instalado más de $200 \mathrm{MW}$ de proyectos de energía renovable.

El parque eólico Lamèque, el primer parque eólico de ACCIONA en la provincia de New Brunswick, puede generar suficiente electricidad libre de emi-

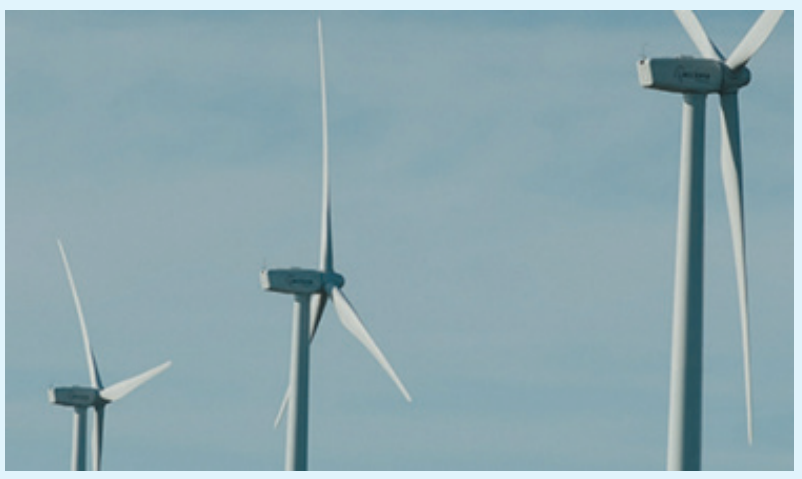

Instantánea: ACCIONA, energía eólica. siones para abastecer a, aproximadamente, 8.000 hogares. El proyecto recibió el apoyo del programa ecoENERGY for Renewable Power del Gobierno de Canadá, con una inversión que alcanza los 13,8 millones de dólares en diez años (ACCIONA Canada, 2021).

Asegurarnos de que tenemos un equilibrio adecuado de energías renovables en la composición de nuestra cartera de energía es esencial. Nuestra asociación con ACCIONA juega un papel importante en el aumento de la diversificación de nuestro mix de generación para incluir más energías renovables. Nos ayuda a alcanzar nuestros objetivos renovables, contribuye a reducir emisiones y respalda nuestra visión de la sostenibilidad: satisfacer las necesidades de hoy y garantizar el mañana. (Gaetan Thomas, presidente y director ejecutivo de NB Power, 2021)

\subsection{Las oportunidades en el sector canadiense de las tecnologías limpias}

\section{Transición energética}

Se espera que el mercado mundial de tecnologías limpias supere los 2,5 billones de dólares en 2022 (Innovation, Science and Economic Development Canada, 2018). La principal oportunidad para los inversores españoles en el sector de las tecnologías limpias es la transición de la industria de recursos naturales hacia alternativas de energía renovable.

Los líderes canadienses en recursos naturales están modernizando su infraestructura de extracción y distribución para adoptar fuentes de energía más limpias, como la eólica y la solar. También impulsan la captura y almacenamiento de carbono y la búsqueda de usos más sostenibles para los desechos, como la utilización de corteza y residuos de los árboles para alimentar turbinas que generan energía limpia. Esto dará lugar a una variedad de oportunidades de inversión en el ciclo de vida del desarrollo de tecnologías limpias, desde startups y prototipos hasta despliegues a gran escala.

Aunque la infraestructura energética de Canadá se ha apoyado tradicionalmente en industrias extractivas como el petróleo y el gas, se puede aprovechar para realizar importantes inversiones en tecnologías limpias y cadenas de suministro asociadas a la producción, distribución y transporte bajos en carbono.

Las oportunidades para las empresas españolas en el campo de las energías renovables se extienden a los grandes sectores canadienses como las manufacturas, la agricultura y el transporte. Las empresas de estos sectores pretenden reducir su huella de carbono $D$ 
mediante la incorporación de energía baja en carbono en sus procesos de producción. Además, el sector inmobiliario de Canadá está impulsando la demanda de tecnologías energéticamente eficientes para gestionar mejor la fuerte demanda de calefacción y de aire acondicionado.

\section{Hidrógeno}

Impulsado por sus múltiples ventajas naturales en la industria del hidrógeno, Canadá se ha posicionado como un líder mundial. Es ya uno de los diez principales productores de hidrógeno a escala mundial. Natural Resources Canada, un departamento del Gobierno federal, afirma que la estrategia de hidrógeno de Canadá podría crear hasta 350.000 empleos verdes de alta calidad durante las próximas tres décadas (Natural Resources Canada,
2020). La producción canadiense de hidrógeno y otras energías limpias resultará cada vez más atractiva para los inversores globales ante las expectativas de aumento de la demanda mundial. Así, mientras el hidrógeno lidera una nueva ola de innovación en tecnologías limpias en todo el mundo, Canadá cuenta con las personas y las tecnologías necesarias para ayudar a las empresas globales a asumir o mantener una posición de liderazgo.

\section{Oportunidades intersectoriales}

Las inversiones en tecnologías limpias también se beneficiarán del creciente apetito por la innovación en sectores líderes como el aeroespacial, la agroindustria y la automoción. Las empresas globales de tecnologías limpias pueden confiar en los recursos humanos de Canadá, altamente formados y capacitados, que $\triangleright$

Nova Bus, integrante del Grupo Volvo, es líder en la provisión de soluciones de transporte público sostenibles en América del Norte. La empresa ha fabricado autobuses eléctricos e híbridos, vehículos de alta capacidad y sistemas de transporte inteligente integrados en Canadá durante veinticinco años.

Nova Bus recibió recientemente 15 millones de dólares de financiación del Fondo de Innovación Estratégica del Gobierno de Canadá (Strategic Innovation Fund) para contribuir a la mejora de sus instalaciones fabriles en Quebec, como parte de una

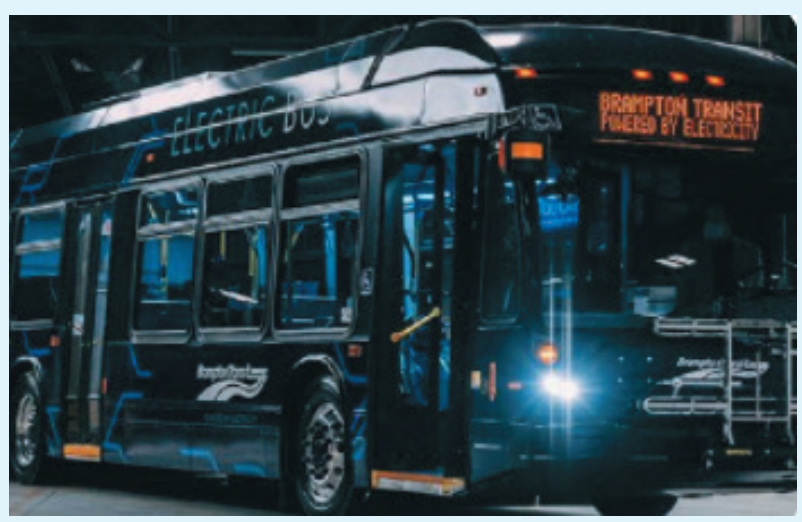
inversión total de casi 185 millones de dólares realizada por la empresa (Nova Bus, 2021). Los fondos se utilizarán también para introducir procesos innovadores de eficiencia energética y tecnologías digitales que aumenten la eficiencia y para apoyar la investigación y el desarrollo hacia el objetivo de autobuses urbanos de cero emisiones.

Estamos muy orgullosos de la asociación con el Gobierno canadiense para colaborar en el innovador sector de fabricación de vehículos de cero emisiones y ayudar a Canadá a alcanzar sus objetivos de sostenibilidad y de una economía más verde. (Martin Lundstedt, director ejecutivo del Grupo Volvo, matriz de Nova Bus, 2021) 
se extienden a todos los sectores y disciplinas, para impulsar la innovación en aplicaciones intersectoriales.

\subsection{Los incentivos para inversores en el sector canadiense de las energías limpias}

Además de una serie de programas de financiación de la innovación y la investigación y el desarrollo, Canadá ofrece una variedad de incentivos y programas para fomentar la inversión en el sector de tecnologías limpias:

- El Centro de Crecimiento Limpio (Clean Growth Hub) ofrece apoyo a empresas y proyectos de energía limpia en todo $\mathrm{Ca}$ nadá, coordinando programas federales y realizando el seguimiento de los resultados de las inversiones federales en tecnología limpia. Su equipo de expertos brinda asesoría a productores y usuarios de tecnologías limpias, ayudándolos a identificar y comprender los programas y servicios más adecuados para sus necesidades.

- El fondo de Tecnología de Desarrollo Sostenible de Canadá (Sustainable Development Technology Canada) invierte 55,1 millones de dólares en proyectos pioneros de tecnologías limpias que lideran la senda de desarrollo de soluciones para afrontar los retos del cambio climático, aire limpio, agua limpia y suelo limpio. El grupo ayuda a las pequeñas y medianas empresas a generar ideas nuevas y sostenibles en los sectores de energía, agricultura y recursos, para que se comercialicen más rápido y las empresas crezcan antes.

\section{El transporte canadiense: el impulso a la demanda de vehículos de cero emisiones}

Para reducir las emisiones de gases de efecto invernadero relacionadas con el transporte, los Gobiernos de todo el mundo están aplicando activamente políticas e incentivos de apoyo a la adopción generalizada de vehículos de cero emisiones. Entre ellos se incluyen vehículos que funcionan con baterías eléctricas, híbridos enchufables o de pila de combustible de hidrógeno.

Canadá está bien posicionado para asumir el liderazgo en los cinco segmentos de la cadena de suministro de baterías para vehículos eléctricos: minería y procesamiento de minerales, fabricación de cátodos y ánodos y precursores químicos, fabricación de baterías, fabricación de vehículos eléctricos y suministro de piezas, y reciclaje de baterías.

\subsection{Las oportunidades en el sector de vehículos de cero emisiones de Canadá}

La expansión del mercado de vehículos de cero emisiones

La Agencia Internacional de Energía pronostica que, para 2030, el stock de vehículos eléctricos alcanzará los 245 millones, un aumento exponencial desde los solo 7,2 millones de 2019. Ello supone un crecimiento promedio anual esperado del 36\% (International Energy Agency, 2020).

Canadá es el único país del hemisferio occidental con capacidad para fabricar vehículos eléctricos de principio a fin. Dispone de la infraestructura y los acuerdos de libre comercio necesarios para acceder directamente a dos de los mercados de vehículos eléctricos más grandes del mundo, Estados Unidos y la UE. 
El sector de vehículos eléctricos de Canadá rebosa de nuevas oportunidades. Ford se ha asociado con el Gobierno de Canadá para adaptar una planta de ensamblaje en Ontario para la fabricación de vehículos eléctricos y baterías. La Asociación de Fabricantes de Componentes de Automoción (Automotive Parts Manufacturer's Association) de Canadá lanzó recientemente el Proyecto Arrow, el primer prototipo de vehículo de cero emisiones, original, construido y plenamente desarrollado en Canadá.

Los inversores españoles en Canadá pueden aprovechar su posición como líder en la tecnología de pilas de combustible de hidrógeno para vehículos pesados, en especial a medida que los municipios canadienses transforman su transporte público y sus flotas hacia vehículos eléctricos de cero emisiones que utilizan hidrógeno y baterías de pilas de combustible. El Gobierno de Canadá invirtió recientemente 600 millones de dólares en el despliegue de la infraestructura para vehículos eléctricos y combustibles alternativos, que soportará el desarrollo de nuevas estaciones de repostaje de hidrógeno en todo el país.

\section{La cadena de suministro desde las minas a la movilidad}

Como respuesta a la mayor demanda de vehículos de cero emisiones, las fábricas de automóviles trabajan rápidamente para construir una nueva cadena de suministro alrededor de la batería de iones de litio, y Canadá está en condiciones de lograrlo.

Canadá es el único país de las Américas con abundancia de las materias primas necesarias para la fabricación de las baterías de iones de litio que se utilizan actualmente en los vehículos eléctricos. Esta disponibilidad de minerales, la infraestructura de fabricación existente y la experiencia canadiense crean enormes oportunidades para los inversores en la industria de vehículos eléctricos en su conjunto, y de las baterías eléctricas en particular.

A escala mundial, Canadá se sitúa entre los principales países del mundo en producción de níquel refinado y está aumentando su capacidad para el procesamiento de elementos de cobalto, grafito, litio y tierras raras, mediante plantas que se despliegan en todo el país. Canadá ocupa actualmente el cuarto lugar en el mundo y el primero en América del Norte por capacidad de materias primas necesarias para la cadena de suministro de baterías, y se espera que ascienda al tercer lugar en 2025 (Mining.com, September 16, 2020). El Plan de Acción Conjunto de Colaboraciones en Minerales Críticos (Joint Action Plan on Critical Minerals Collaborations), firmado entre Canadá y Estados Unidos en 2020, facilitará el suministro fiable de minerales críticos en ambos lados de la frontera.

Este ecosistema dinámico abre muchas puertas para Canadá como líder en el suministro directo de materias primas a los fabricantes de cátodos y ánodos. También existe potencial para los productores y proveedores canadienses de elementos clave de la batería, como los cátodos, que representan más de la mitad del coste de fabricación de los vehículos, o incluso para la producción de baterías por sí mismos, como un siguiente paso de valor añadido en la cadena de suministro de baterías.

Canadá es el duodécimo mayor productor de vehículos global, pero asciende al quinto puesto a nivel mundial en la producción de vehículos comerciales - con casi 1,4 millones de vehículos producidos en 2018 (Sharpe, Lutsey, Smith y Kim, 2020)—. New Flyer, Nova Bus, Lion Electric Company, GreenPower Motor Company, Grande West y BYD fabrican $D$ 
autobuses de cero emisiones en Canadá. General Motors, Ford Motor Company, Toyota y Fiat Chrysler se han comprometido a fabricar vehículos eléctricos en Canadá. El país también está equipado con un ecosistema vibrante, compuesto por más de 700 suministradores de repuestos para automóviles, muchos de los cuales ya suministran repuestos para vehículos híbridos y de batería eléctrica.

Como líder de América del Norte en reciclaje de baterías, Canadá se encuentra en una posición privilegiada para completar la cadena de suministro y ofrecer a los mercados globales un enclave sostenible para reciclar baterías y asegurar una fuente estable de materiales reciclados. Para los inversores españoles en Canadá esto supone una oportunidad ideal para facilitar un reciclaje seguro y sostenible que cumpla con los requisitos del mercado europeo y pueda contribuir a cumplir el objetivo de neutralidad climática del Pacto Verde Europeo. También surge una fuente estable de metales reciclados para la futura producción de baterías en Canadá.

\subsection{Los incentivos para inversores en el sector de vehículos de cero emisiones}

Además de una serie de programas de financiación de innovación e investigación y desarrollo, Canadá ofrece también incentivos que impulsarán el crecimiento en el sector de vehículos de cero emisiones.

El Programa de Infraestructura de Vehículos de Cero Emisiones (Zero Emission Vehicle Infrastructure Program) es un programa de $280 \mathrm{mi}-$ llones de dólares en cinco años, que tiene como objetivo electrificar los vehículos ligeros de Canadá y cambiar a combustibles más limpios. El programa apoya la infraestructura que ampliará la disponibilidad de estaciones de carga y repostaje de vehículos eléctricos en Canadá. Aumentará la disponibilidad de instalaciones de carga y de repostaje de hidrógeno, localizadas donde los canadienses viven, trabajan y juegan.

\section{Otras razones para elegir Canadá}

Aunque hemos esbozado posibilidades en tres sectores, el compromiso de Canadá con la innovación está impulsando el crecimiento en todos los sectores, incluidos el aeroespacial, la fabricación avanzada y las ciencias de la vida. Las ventajas de Canadá como destino de inversiones se extienden a todas las empresas globales y sus empleados.

\section{La facilidad de hacer negocios}

Canadá ocupa el primer lugar entre los países del G20 que ofrecen el mejor entorno empresarial para el periodo 2019 a 2023 y es también reconocido como el lugar donde es más fácil crear una empresa.

Canadá tiene la tasa impositiva marginal efectiva más baja del G7, bajos costes operativos, así como una serie de créditos fiscales y programas de incentivos a la inversión. Canadá proporciona acceso a la financiación mediante deuda y acciones tanto a través de mercados organizados como de grandes inversores institucionales.

\section{Estabilidad económica y calidad de vida}

Canadá es conocido en todo el mundo por su estabilidad económica y política, modestas fluctuaciones del valor de la divisa y de los tipos de interés, y resiliencia en tiempos de incertidumbre. La previsibilidad económica del $\triangleright$ 
país representa una ventaja estratégica sobre los mercados emergentes, donde las variables de bajo coste conllevan primas de alto riesgo. Es una sociedad estable con Estado de derecho, capacidad para proteger la propiedad intelectual y una gobernanza eficaz y predecible.

Un sistema de transporte consolidado

Canadá ofrece a los inversores globales una infraestructura de transporte fiable y consolidada y cadenas de suministro nacionales e internacionales interconectadas para ayudar a los inversores globales a llegar a los mercados internacionales. Canadá tiene 24 aeropuertos internacionales, 17 puertos marítimos y 117 cruces fronterizos con Estados Unidos.

\section{Obtenga más información de la agencia de promoción y atracción de inversiones de Canadá}

¿Tiene interés en explorar cómo Canadá puede contribuir a que su empresa se expanda y prospere? Invest in Canada es la agencia de promoción y atracción de inversiones global de Canadá. Ofrecen servicios personalizados para ayudar a las empresas globales a desbloquear oportunidades de inversión en Canadá. Invest in Canada trabaja directamente con inversores globales para proporcionar un punto de contacto único y fluido para ayudar a las empresas globales que buscan establecer operaciones, expandir sus empresas o combinar fuerzas con organizaciones canadienses.

\section{Bibliografía}

ACCIONA Canada. (2021). World leader in renewable energy. https://www.acciona.ca/business-divisions/energy/
Agriculture and Agri-Food Canada. (2020). Overview of the Canadian agriculture and agri-food sector 2018. https://agriculture.canada.ca/en/ canadas-agriculture-sectors/sector-overviewsdata-and-reports/overview-canadian-agricultureand-agri-food-sector-2018

Biospringer by Lesaffre. (2021). The 3 reasons why consumers crave meat analogues. https://biospringer.com/en/why-consumers-crave-meatanalogues/

Canada Action. (2021). Canada Ranks 2nd on the 2021 Global Cleantech Innovation Index. https:// www.canadaaction.ca/cleantech-innovationindex-ranking

Department of Finance Canada. (2019). Canada Has Lowest Tax Rate on New Business Investment in G7. https://www.canada.ca/en/department-finance/news/2019/07/canada-has-lowesttax-rate-on-new-business-investment-in-g7.html

Employment and Social Development Canada. (2021a). Program requirements for the Global Talent Stream. https://www.canada.ca/en/employment-social-development/services/foreign-workers/global-talent/requirements.html

Employment and Social Development Canada. (2021b). Global Skills Strategy. https://www.canada.ca/en/employment-social-development/ campaigns/global-skills-strategy.html

Employment and Social Development Canada. (2021c). Hire a top foreign talent through the Global Talent Stream. https://www.canada.ca/en/ employment-social-development/services/foreign-workers/global-talent.html

Export Development Canada. (2019). Cream of the crop: Canada's agri-food sector poised for growth. https://www.edc.ca/en/article/canadasagri-food-sector.html

Export Development Canada. (2020). Canada's Cleantech Future Looks Bright. https://octia.ca/ wp-content/uploads/2021/03/EDC_canadacleantech-future.pdf

Global Affairs Canada. (2020). CETA explained. https://www.international.gc.ca/trade- $D$ 


\section{CANADÁ: UN PAÍS DE OPORTUNIDADES PARA LA INVERSIÓN EXTRANJERA...}

commerce/trade-agreements-accords-commerciaux/agr-acc/ceta-aecg/ceta_explained-aecg_ apercu.aspx?lang=eng

Government of Canada. (2019). Sous vide slowcooker innovates with Spanish flair in Canada. https://www.international.gc.ca/world-monde/ stories-histoires/2019/CETA-cascajares. aspx?lang=eng

Government of Saskatchewan. (2020). Agriculture and Agri-Value. https://www.saskatchewan.ca/ business/investment-and-economic-development/key-economic-sectors/agriculture-andagri-value

Immigration, Refugees and Citizenship Canada. (2019). Second Anniversary of the Global Skills Strategy. https://www.canada.ca/en/immigrationrefugees-citizenship/news/2019/06/secondanniversary-of-the-global-skills-strategy.html

Innovation, Science and Economic Development Canada. (2018). Report from Canada's Economic Strategy Tables: Clean Technology. https:// www.ic.gc.ca/eic/site/098.nsf/eng/00023.html

International Energy Agency. (2020). Global EV Outlook 2020. https://www.iea.org/reports/global-evoutlook-2020

Invest in Canada. (2021). Canada's Free Trade Agreements. https://www.investcanada.ca/programsincentives/canadas-free-trade-agreements

KPMG. (2020). Advantage Canada: Reshaping Supply Chain Investment Opportunities After COVID-19. Invest in Canada.

Mining.com (September 16, 2020). Canada ranked 4th, US 6th in lithium-ion battery supply chain. https://www.mining.com/new-ranking-hascanada-4th-us-6th-in-lithium-ion-battery-supplychain/

National Research Council Canada. (2019). Plantbased protein market: global and Canadian market analysis. https://nrc.canada.ca/en/researchdevelopment/research-collaboration/programs/ plant-based-protein-market-global-canadianmarket-analysis
Natural Resources Canada. (2019). Canadian Mineral Production. https://www.nrcan.gc.ca/sciencedata/science-research/earth-sciences/earthsciences-resources/earth-sciences-federalprograms/canadian-mineral-production/17722\#s3

Natural Resources Canada. (2020). The Hydrogen Strategy for Canada. https://www.nrcan.gc.ca/ climate-change/the-hydrogen-strategy/23080

Nova Bus. (2021). Nova Bus continues to invest in its growth, innovation and technology with the support of the Government of Canada. https:// novabus.com/blog/2021/06/08/novabus-continues-to-invest-in-its-growth-innovationand-technology-with-the-support-of-the-government-of-canada/

OECD. (2020). Education at a Glance. https://www. slideshare.net/OECDEDU/education-at-a-glance2020-global-insights

Research and Markets. (2019). Global Plant-based Protein Industry Report 2019-2025 - Industry was Valued at \$18.5 Billion in 2019, and is Projected to Reach $\$ 40.6$ Billion by 2025 . https:// www.globenewswire.com/news-release/ 2019/12/17/1961432/0/en/Global-Plant-basedProtein-Industry-Report-2019-2025-Industrywas-Valued-at-18-5-Billion-in-2019-and-is-Projected-to-Reach-40-6-Billion-by-2025.html

Sharpe, B., Lutsey, N., Smith, C., and Kim, C. (2020). Power Play: Canada's Role in the Electric Vehicle Transition. White Paper, april 2020. International Council on Clean Transportation. https:// theicct.org/sites/default/files/publications/CanadaPower-Play-ZEV-04012020.pdf

Statistics Canada. (2020). Environmental and clean technology products sector grew at twice the pace as the total economy in 2019. https:// www150.statcan.gc.ca/n1/daily-quotidien/201218/ dq201218d-eng.htm

Statistics Canada. (2021). Postsecondary enrolments, by International Standard Classification of Education, institution type, Classification of Instructional Programs, STEM and BHASE groupings, status of student in Canada, age group and gender. https://www150.statcan.gc.ca/t1/tbl1/en/ tv. action?pid=3710016301 
